\title{
Analog of the Peierls-type phase transition in nuclear matter
}

\author{
István Lovas, Lívia Molnár, Kornél Sailer \\ Department for Theoretical Physics, Lajos Kossuth University, Debrecen, Hungary \\ Walter Greiner \\ Institut für Theoretische Physik, Johann Wolfgang Goethe Universität, Frankfurt am Main, Germany \\ Received 17 November 1993 \\ Editor: R. Gatto
}

\begin{abstract}
A phase transition of nuclear matter into a periodic phase has been found. Studying the properties of this phase transition an analogy has been observed with the Peierls' transition taking place in quasi-one-dimensional electric conductors.
\end{abstract}

Recently we have found a periodic structure in nuclear matter [1] in the framework of quantumhadrodynamics using mean-field approximation [2,3]. Studying the temperature dependence of this periodic structure we have observed an analogy with the Peierlstype phase transition [4-6].

In this letter we summarise the main features of the periodic phase of nuclear matter and we make a comparison with the periodic structure observed in quasi-one-dimensional electric conductors.

In the last few years the possibility of a periodic structure in nuclear matter was investigated extensively [7-9]. In these works the periodic behaviour of the matter density was studied. In our recent work we have found a periodic structure in the current density at constant matter density.

In the framework of the quantumhadrodynamics the nucleons $(\psi)$, the scalar mesons $(\sigma)$, and the vector mesons $\left(\omega^{\mu}\right)$ are described by the following field equations:

$$
\left[\gamma_{\mu}\left(i \partial^{\mu}-g_{\omega} \omega^{\mu}\right)-\left(m-g_{\sigma} \sigma\right)\right] \psi(x)=0
$$

$$
\begin{aligned}
& \square \sigma+m_{\sigma}^{2} \sigma=g_{\sigma} \bar{\psi} \psi, \\
& \square \omega_{\mu}+m_{\omega}^{2} \omega_{\mu}=g_{\omega} \bar{\psi} \gamma_{\mu} \psi
\end{aligned}
$$

The coupling constants $g_{\omega}, g_{\sigma}$ and the masses $m, m_{\sigma}$, $m_{\omega}$ are given in [1]. In the mean field approximation the meson field operators are replaced by their expectation values, which are assumed to have the following forms:

$$
\begin{aligned}
& \langle\sigma(x)\rangle=\bar{\sigma}, \quad\left\langle\omega^{0}(x)\right\rangle=\bar{\omega}^{0}, \\
& \left\langle\omega^{1}(x)\right\rangle=\bar{\omega} \cos k z, \quad\left\langle\omega^{2}(x)\right\rangle=-\bar{\omega} \sin k z, \\
& \left\langle\omega^{3}(x)\right\rangle=0 .
\end{aligned}
$$

According to this Ansatz the $\omega$-field has a static, periodic variation along the $z$ axis, defined by the wave vector $\boldsymbol{k}$. This periodicity induces a similar periodic behaviour of the nuclear vector current, i.e. the matter density $\left\langle\bar{\psi} \gamma^{0} \psi\right\rangle$ is constant and the current densities $\left\langle\bar{\psi} \gamma^{1} \psi\right\rangle$ and $\left\langle\bar{\psi} \gamma^{2} \psi\right\rangle$ are periodic.

In this approximation the single particle nucleon states can be considered as independent quasiparticle states described by Bloch waves: 
$\psi_{i}(p, x)=e^{-i p x} \sum_{n=-N}^{N} u_{i}(p, n) e^{-i n k z}$.

Here the bispinors $u_{i}(p, n)$ are the solutions of the following equation

$$
\sum_{n^{\prime}=-N}^{N} H_{n n^{\prime}} u_{i}(\boldsymbol{p}, n)=E_{i} u_{i}(\boldsymbol{p}, n),
$$

where

$$
\begin{aligned}
H_{n n^{\prime}} & =\gamma^{0}\left(\gamma \boldsymbol{p}+\gamma^{3} n k+\left(m-g_{\sigma} \bar{\sigma}\right)+\gamma^{0} g_{\omega} \bar{\omega}^{0}\right) \delta_{n n^{\prime}} \\
& -\frac{1}{2} g_{\omega} \bar{\omega} \gamma^{0} \\
& \times\left[\left(\gamma^{1}-i \gamma^{2}\right) \delta_{n+1, n^{\prime}}+\left(\gamma^{1}+i \gamma^{2}\right) \delta_{n-1, n^{\prime}}\right] .
\end{aligned}
$$

The source terms $\bar{\psi} \Gamma \psi$ are also replaced by their expectation values $\langle\bar{\psi} \Gamma \psi\rangle$, which are defined by the help of the Fermi-Dirac distribution:

$$
\left(1+\exp \left\{\frac{E_{i}(\boldsymbol{p})-\mu}{T}\right\}\right)^{-1},
$$

where $E_{i}(\boldsymbol{p})$ are the energy eigenvalues from Eq. (6). The temperature and the baryon chemical potential are denoted by $T$ and $\mu$, respectively. (The chemical potential of the antibaryons is $-\mu$.) The field equations lead to a set of selfconsistent equations. At fixed values of the parameters $T, \mu$ and $k$ these selfconsistent equations can be solved numerically for $\bar{\sigma}, \bar{\omega}^{0}$ and $\bar{\omega}$.

From the solutions the following conclusions can be drawn by stability analysis:

(i) At low densities periodic solutions $(\bar{\omega} \neq 0)$ exist. They are, however, instable due to a saddle point of the thermodynamical potential $\Omega$ defined as

$$
\Omega=-T \ln \{\operatorname{Tr} \exp (-[\hat{H}-\mu \hat{B}] / T)\},
$$

where the Hamiltonian and the baryon number operator are denoted by $\hat{H}$ and $\hat{B}$, respectively. Above a critical nucleon density $\rho_{c}$ the periodic solutions become stable due to a true minimum of the thermodynamical potential $\Omega$.

(ii) The system is anisotropic which can be characterised by the quantity $A$ defined as

$$
A=\frac{P_{\|}-P_{\perp}}{P_{\|}+P_{\perp}}
$$

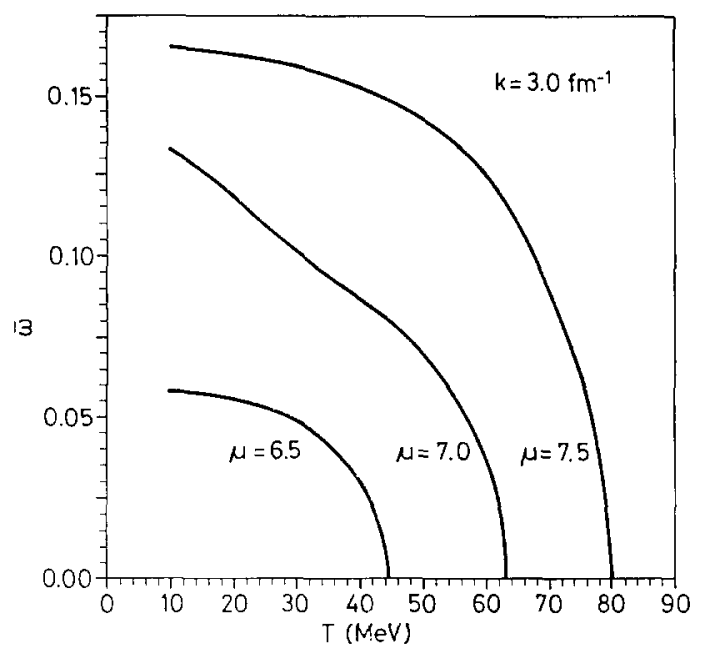

Fig. 1. The amplitude $\bar{\omega}$ of the periodic component of the $\omega$-meson field as the function of the temperature $T$ for the wave number $k=3.0 \mathrm{fm}^{-1}$. The parameter of the curves is the baryon chemical potential $\mu$.

where the pressure parallel and perpendicular compared to $k$ are denoted by $P_{\|}$and $P_{\perp}$, respectively. This means that the periodic structure of the nuclear matter can only exist if an appropriate anisotropic external pressure is exercised on it. In thermodynamical equilibrium assuming an isotropic external pressure only the "trivial" $(\bar{\omega}=0)$ solution exists.

(iii) Changing the temperature $T$ at fixed values of $\mu$ and $k$ a critical temperature $T_{c}$ can be reached. Above $T_{c}$ there are no periodic solutions, i.e. only the "trivial" $(\bar{\omega}=0)$ solution exists. Below the critical temperature $T_{c}$ the $\omega$-mesons with wave vector $\boldsymbol{k}$ begin to condensate. The order parameter of this phase is the amplitude of the static, periodic $\omega$-field which, is identical with the self consistent solution of the mean field equations $\bar{\omega}$. The situation is similar to the pion condensation $[14,15]$. On Fig. 1. the temperature dependence of the order parameter $\bar{\omega}$ is shown at a fixed value of $k\left(k=3.0 \mathrm{fm}^{-1}\right)$ and fixed values of the baryon chemical potential $\mu$.

(iv) On Fig. 2. the critical density $\rho_{c}(k)$ is shown as a the function of $k$. As it is seen the $k$ dependence of $\rho_{c}(k)$ is linear. To understand this behaviour we assume that the Fermi surface of the system can be considered as a cylinder. (In 


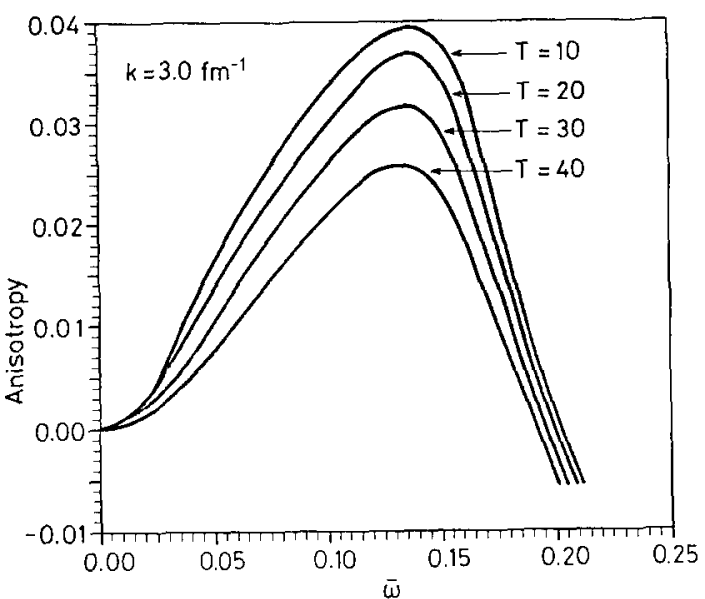

Fig. 2. The critical density $\rho_{c}(k)$ as the function of the wave number $k$.

the reality it is only cylindrically symmetric, but not a cylinder.) Then the baryon density can be expressed as follows

$$
\rho_{c}(k)=k_{F \perp}^{2} k_{F \|} / \pi^{2},
$$

where the Fermi momenta parallel and perpendicular to the wave vector $k$ are denoted by $k_{F \perp}$ and $k_{F \|}$, respectively. In this approximation the density is a linear function of $k_{F \|}$. Consequently there is a linear relation between $k$ and $k_{F \|}$.

Looking for an explanation for the enumerated features of the periodic phase, we have found an analogy in solid state physics. It was pointed out by Kohn [6], in 1959 that the response function $F$ of a free electron system, defined by

$$
\begin{aligned}
F(q) & =\frac{1}{\pi a_{0} q^{2}} \\
\times & {\left[1+\frac{k_{F}}{q}\left(1-\frac{q^{2}}{4 k_{F}^{2}}\right) \ln \left(\left|\frac{q+2 k_{F}}{q-2 k_{F}}\right|\right)\right], }
\end{aligned}
$$

has an anomaly at $q=2 k_{F}$, where the Fermi momentum and the Bohr radius are denoted by $k_{F}$ and $a_{0}$, respectively. The derivative of $F(q)$ has a logarithmic singularity at this point. In a metal the phonons interacting with the electrons induce a change of the electron density, which is enhanced in the vicinity of the singularity. As a response to this the frequency of the phonons $\omega(q)$ for $q \approx 2 k_{F}$ decreases. This can be observed in inelastic neutron scattering. There exists a critical temperature $T_{c}$ where the phonon fre- quency $\omega\left(2 k_{F}\right)$ vanishes $[5,4]$. At this point there is a phase transition, where a static periodic deformation of the crystall lattice begins to develop. This Peierlstype phase transition occurs predominantly in quasione-dimensional systems, where the electronic excitation with $q=2 k_{F}$ is associated with a sufficiently large phase space volume. The discovery of the quasi-onedimensional systems like the TCNQ and KCP salts [10] opened an astonishingly rich realm of phenomena of charge density and spin density waves associated with the Peierls transition. It is worth of mentioning that the Peierls transition is also favoured for threedimensional systems with Fermi surface of cylindrical shape, as shown by Afanasev and Kagan [11].

In our case the periodic structure is present in the current densities and consequently in the spin density. Therefore it is related to the spin density waves of quasi-one-dimensional conductors [12,13].

Summarising, in nuclear matter a phase-transition occurs if an adequate anisotropic external pressure is exercised on the system. This kind of anisotropy can be produced in heavy ion reactions or in astrophysical objects by gravitational force. The phase transition of nuclear matter under anisotropic external pressure from the normal phase to the periodic one is the analog of the Peierls-type phase transition occuring in quasione-dimensional systems.

The authors are very much indebted to A. Virosztek for the illuminating discussions. Part of the calculations were performed on the Microvax computer donated by the Alexander von Humboldt Foundation.

\section{References}

[1] I. Lovas, L. Molnár, K. Sailer and W. Greiner, Phys. Rev. C 45 (1992) 1693.

[2] J.D. Walecka, Ann. Phys. (N.Y.) 83 (1974) 491.

[3] S.D. Serot and J.D. Walecka, Adv. Nucl. Phys. 16 (1986) I

[4] H. Fröhlich, Proc. Roy. Soc. A 223 (1954) 296.

[5] R.E. Peierls, Quantum theory of solids (Oxford University Press, London, 1955) p. 108.

[6] W. Kohn, Phys. Rev. Lett. 2 (1959) 393.

[7] Yu.B. Ivanov, Nucl. Phys. A 474 (1987) 669

[8] B.L. Friman and P.A. Henning, Phys. Lett. B 206 (1988) 579.

[9] C.E. Price, J.R. Shepard and J.A. McNeil, Phys. Rev. C 41 (1990) 1234; C 42 (1990) 247. 
[10] Low-dimensional co-operative phenomena. The possibility of high-temperature superconductivity, ed. H.J. Keller (Plenum Press, New York, 1977).

[11] A.M. Afanasev and Y. Kagan, Zh. Eksp. Teor. Fiz. 43 (1962) 1456 (Sov. Phys. JETP 16 (1963) 1030).

[12] G. Gruner, Density Waves in Solids, Lecture Notes on Physics ( to be published).
[13] K. Maki and A. Virosztek, Phys. Rev. B 41 (1990) 557. [14] A.B. Migdal, Zh. Eksp. Teor. Fiz. 61 (1971) 2209.

[15] I. Lovas, J. Németh and K. Sailer, Nucl. Phys. A 430 (1984) 731. 